\title{
A Personalized Exercise Trainer for Elderly
}

\author{
Daniel Steffen*, Gabriele Bleser*, Markus Weber*, Didier Stricker* \\ ${ }^{*}$ German Research Center for Artificial Intelligence (DFKI) GmbH \\ Department Augmented Vision \\ Kaiserslautern, Germany \\ \{firstname.lastname\}@dfki.de
}

\author{
Laetitia Fradet ${ }^{\dagger}$, Frédéric Marin ${ }^{\dagger}$ \\ †Université de Technologie de Compiègne \\ UMR6600-Biomécanique et Bioingénérie \\ Compiègne, France \\ \{firstname.lastname\}@utc.fr
}

\begin{abstract}
Physical activity provides many physiological benefits. On the one hand it reduces the risk of disease outcomes. On the other hand it is the basis for proper rehabilitation in case of or after a severe disease. Both aspects are especially important for the elderly population. Within this context, the present paper proposes a personalized, home-based exercise trainer for elderly people. The system is based on a wearable sensor network that enables capturing the user's motions. These are then evaluated by comparing them to a prescribed exercise, taking both exercise load and technique into account. Moreover, the results are translated into appropriate feedback to the user to assist the correct exercise execution. A novel part of the system is the generic personalization by means of a supervised teach-in phase.
\end{abstract}

Index Terms-physical activity monitoring, home-based rehabilitation, HCI, wearable sensors, health promotion, personalization.

\section{INTRODUCTION}

Physical activity provides many physiological benefits, reduces the risk of disease outcomes, and generates important psychological gains [1]. The nature of physical activity can be categorized as aerobic activity promoting cardiovascular fitness or strength exercises promoting musculoskeletal fitness. Particularly in frail population, balanced activity of both types is important for keeping functional independence [1]. It is then essential to promote the practice of physical activity especially at home since it has been shown that adherence to exercise is greater when performed at home than when performed in centers [2]. However, this physical activity has to be supervised in order to improve physical fitness whilst minimizing the risk of an overuse injury [3]. Moreover, this supervision of physical activity has to be achieved whilst providing feedback to the user regarding his activity. This preserves or increases motivation and program adherence.

The supervision of physical activity at home has been until now mainly related to rehabilitation follow-up. Information and Communication Technologies (ICT) have been used for that purpose by providing rehabilitation services at home over telecommunication networks and the internet. This constitutes the recent field of telerehabilitation [4]. However, only a few of these projects have tried to elaborate a more complete approach of services including a monitoring of physical activity, a wellness diary, mentoring sessions, and a web-portal to facilitate personal goal setting and to assess the progress of each patient in the program [5]. Additionally, most often these telerehabilitation projects focus on a specific aspect of physical activity related to a special disease or pathology and do not consider physical activity as a whole.

The aim of the present project is to promote physical activity especially in elderly by creating a platform that can supervise and help the practice of physical activity. This platform can also be used as a support of home-based physical rehabilitation. An overview of the overall system with its different configurations, functionalities and services is presented in Section III.

This paper focuses on the physical activity monitoring aspects. As previously mentioned, two different categories of physical activity are recommended: aerobic and strength training activities. Recently developed methodologies based on inertial sensors have been proposed to monitor aerobic activities [6], [7]. With such methodologies it is possible to control, whether the F.I.T.T. (Frequency, Intensity, Type, and Time of training) principles of training [8] are well respected by the subject. A similar approach has already been implemented for the present project [9].

During strength training, to our knowledge, no method has been proposed. Current video games include feedback provided by accelerometers in order for users to follow some fitness exercises, but only few parameters are taken into account and the proposed methods are not documented. Studies have shown that strength training can result in injuries when exercise technique is not respected [8]. Now, the number of these injuries and the damages caused by these injuries might increase in both frailer population and population not used to practice strength training such as elderly or pathological population. Therefore, an appropriate monitoring of strength training has to be proposed. This work presents a methodology for this monitoring, describing design, implementation, and evaluation of respective hardware and software infrastructures, algorithms and applications.

\section{Use CASe: Personalized exercise trainer}

The overall system (cf. Section III) can be used in different configurations providing a broad set of functionalities and services. This paper, however, focuses on the special use case of the system as personalized exercise trainer, in particular when performing unusual exercises. This personalized trainer has then to help the subject to follow a physical activity program and to perform the recommended exercises correctly. 


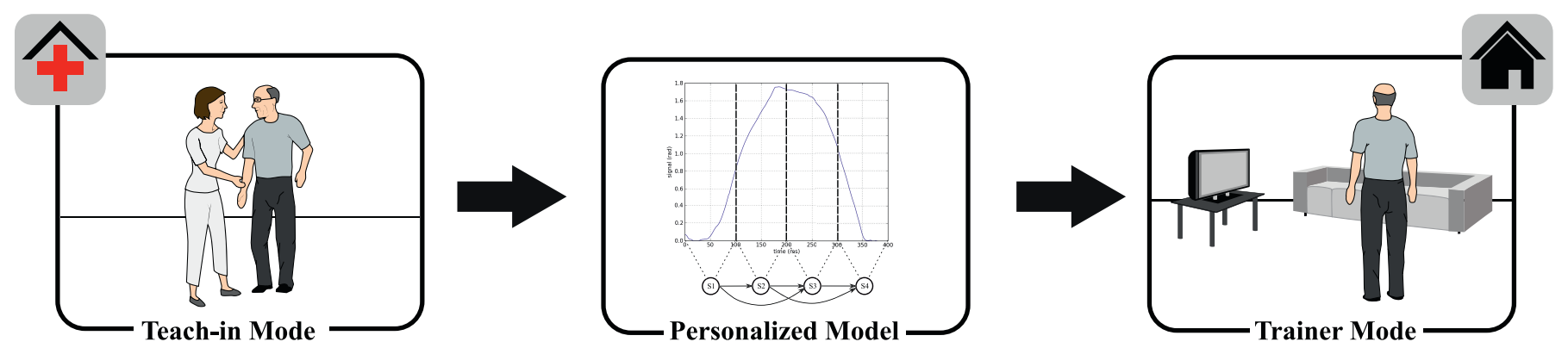

Fig. 1: Overview of the personalized exercise trainer use case. A reference motion is recorded during a guided teach-in phase. From this a personalized model is generated and then used in the trainer mode at home as evaluation reference.

Within this context, data collection, processing and presentation are detailed putting emphasis on two major aspects: (a) Usability for elderly: As argued in the previous section physical activity and particularly strength training have to be promoted within the elderly population but the incorrect execution of exercises could occasion more damages than benefits. (b) Personalization: Personalized training implies to respect limited mobility of the individual subject during the training and automatic exercise evaluation. This is particularly important for the elderly population, where a higher percentage of limiting pathologies is to be expected.

The basic idea for enabling personalized exercise training support is depicted in Figure 1. The aim is to accurately evaluate the movement performed by the user and, based on this, to provide valuable feedback in real-time. In short, the aim is to help the user to perform the training correctly. However, what is correct depends on the limitations and goals of the individual user. Hence, in order to achieve personalization in a generic way, the idea is to create a personalized reference for evaluation within a first supervised training session (see Figure 1). During this session, the proposed system is used in a teach-in mode. While the patient is shown how to perform the exercises according to his special needs, inertial data are recorded and preprocessed (cf. Section IV). The estimated motion sequences are further processed to derive a personalized model for each exercise (cf. Section V-A). At home, the patient uses the system in the trainer mode. In this mode, the performed motion is evaluated by comparing it to the stored reference models (cf. Section V-B). The evaluation results are translated into helpful audio and visual feedback for the user. The user interface also provides a guided exercise preparation and a summary at the end (cf. Section VI).

\section{SySTEM OVERVIEW}

The overall system is modular and flexible: It is composed of four self-contained components that communicate with each other over network using standard and customized protocols. The individual components (data collection, data processing, data presentation, and data management) are outlined in the following.

The data collection component is based on a network of (wireless) sensors and a mobile processing unit that are worn by the user. The data collection allows measuring his motions and vital signs. The complete set-up comprises up to ten miniature inertial sensors and complementary sensors, such as a heart rate monitor. Preprocessing includes correction, filtering and synchronization of the raw sensor data, as well as, the derivation of higher-level information, for instance, the estimation of body posture in terms of joint angles from the data of several body-mounted inertial sensors.

The data processing component consists in softwares for analyzing and characterizing the physical activity of the user based on the preprocessed data. Several individual algorithms have been developed for enabling sophisticated analysis. As indicated in the introduction, these range from the derivation of the general F.I.T.T. parameters to the accurate evaluation of strength exercises, the latter being in the focus of the present paper. This way, the data processing component supports a holistic way of physical activity analysis.

The data presentation component is a web application for physical activity visualization, guidance and feedback. Individual user interfaces have been developed for presenting the different dimensions of physical activity as described above. Particular effort has been spent in developing the trainer mode. In this mode, the user gets instructions on how to prepare for and perform special strength exercises, such as biceps curls or push-ups. During training, he receives audio and visual feedback on the way he executes the training program. This feedback is based on the evaluation of the performed motion as delivered by the data processing component. After the training session, the user gets a summary of the exercise quality. This enables self-monitoring and can motivate him to increase his performance in the next session.

The data management component is based on a web application for collecting and managing all relevant information in an electronic health record (EHR). Apart from physical activity data, this web application also includes a comprehensive summary of a medical record, a rehabilitation plan management module and a health status survey module. This component extends the system for usage as a remote patient monitoring system. Healthcare professionals are facilitated in maintenance of an EHR of their patients and in the establishment and follow up of personalized rehabilitation plans. In turn, the patients, who can gain access via web or on interactive TV (i-TV) 


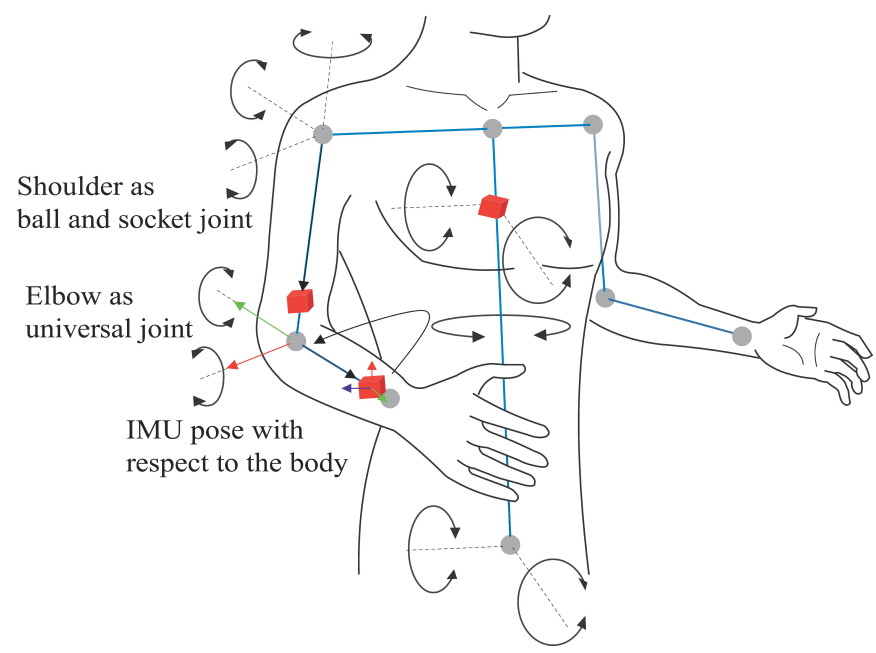

Fig. 2: Functional upper body model with indicated IMU placement.

interface, are provided with the means to stay in touch with their healthcare professionals. The i-TV solution is used to increase the acceptance and ease the learning amongst elderly users, who generally are not very familiar with computers.

The following sections describe the design, algorithms, implementation, and evaluation of the components that make up the personalized exercise trainer.

\section{BODY SENSOR NETWORK AND MOTION ESTIMATION}

In order to accurately evaluate the exercises whilst being performed, precise body motion estimation is required in realtime. The section describes how this information is deduced from the data of inertial measurement units (IMUs) attached to the body.

\section{A. Inertial measurement units (IMUs)}

Two generations of IMUs have been developed in close cooperation with the company Trivisio [10]. The first generation wired sensors are lightweight (22 $\mathrm{g}$ without cable) and small $(30 \times 30 \times 13 \mathrm{~mm})$. The latest wireless generation has, due to the integrated battery (10 hours operating time), an increased weight of $48 \mathrm{~g}$ and dimensions of $56 \times 42 \times 19 \mathrm{~mm}$. Each IMU contains a 3-axis MEMS accelerometer and gyroscope, and a 3-axis magneto-inductive magnetic sensor, all sampled at $100 \mathrm{~Hz}$.

\section{B. Inertial body posture estimation}

The pose and motion of the body are contained in the measured accelerations, angular velocities, and magnetic fields from the IMUs attached to it. These measurements are compared to predictions based on a biomechanical body model. The pose kinematics are then determined using model based sensor fusion. A drawing of the IMUs in relation to the upper body model is depicted in Figure 2.

The biomechanical model determines the degrees of freedom (DOF), and based on this, the set of movements that can be captured. In the context of the proposed system, the model is a compromise between simplicity and accuracy: It is detailed enough to provide accurate data for the exercise evaluation and user feedback. At the same time it enables an efficient real-time estimation. The complete model is a functional model consisting of ten rigid bodies (torso, pelvis, upper arms, forearms, upper legs, and lower legs) connected by anatomically motivated restricted joints. The model is parameterized by the segment lengths, so that it can be personalized for the individual subject.

At least one IMU is required at each segment that should be monitored in order to obtain its complete orientation. For an accurate capturing of the whole body pose, ten sensors are needed. The sensors' placement is indicated in Figure 2. It is chosen with respect to several constraints: it should be unobtrusive, limit the skin and muscles motion artifacts, and ensure an easy positioning and accurate data. Therefore, IMUs are placed at bones, ligaments, and between muscles.

In order to obtain easy, fast and repeatable positioning on the body, two prototypical fixation methods have been developed for the upper extremities and are currently tested: (a) a jacket with integrated sensors, (b) velcro straps with clippers for fixation above the normal cloths (see Figure 3).

To be able to use the IMU measurements for estimating the body pose, the poses of the sensors, more specifically, their orientations relative to the body segments must be calibrated. For this, an easy to perform calibration procedure based on IMU measurements taken under known static poses [11] has been developed. The devised procedure requires the user to stand upright with the arms straight down and the thumbs forward, and then bent over.

The estimation of the body posture in terms of joint angles, angular rates and accelerations is carried out by a set of nested extended Kalman filters (EKFs) [12]. The configuration of the limbs (arms, legs) and the trunk segments are each estimated in an individual EKF. The EKFs are then loosely coupled. The measurement equations are based on forward kinematic equations and the functional model presented above. Given

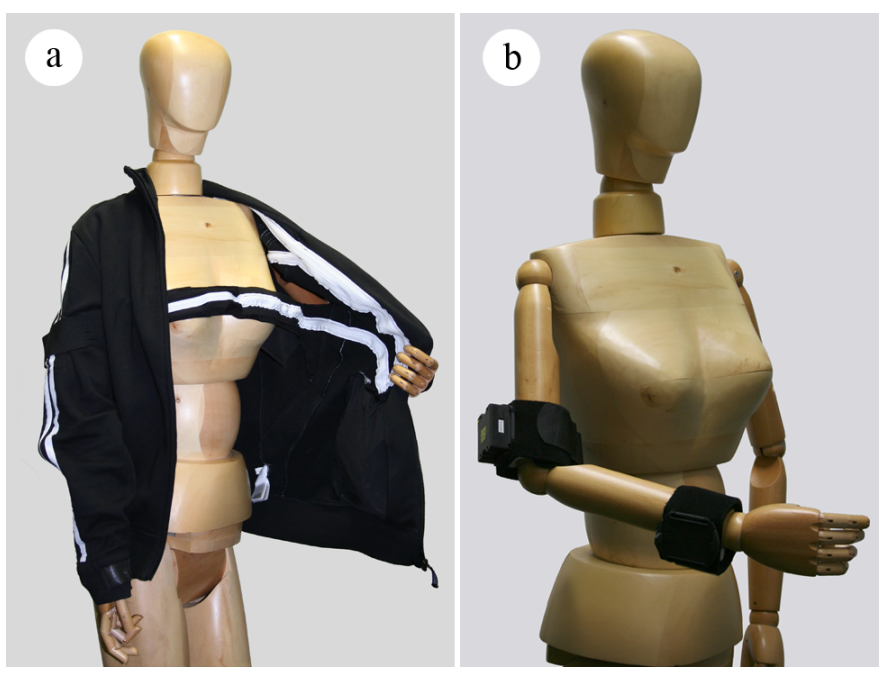

Fig. 3: Sensor fixation: sensor jacket (a) and velcro straps (b). 
the joint angles from the filter and the model, the body pose is fully determined. The algorithm is described in more detail in [13].

\section{Implementation and evaluation}

The method for body motion estimation is implemented in a modular, self-contained $\mathrm{C}++$ software component, which is decoupled from the low-level IMU communication and, hence, independent from the actual IMU hardware. The software automatically identifies the connected sensors and tracks the respective parts of the body at $100 \mathrm{~Hz}$. Besides local data logging functionality, it provides a bidirectional network interface for receiving commands and sending off the processing results with minimal delay. For this, a customized protocol and binary message format has been developed.

The accuracy of the body tracking system has been evaluated against a validated optoelectronic motion capture system [14] in combination with a validated biomechanical model [15]. The results are detailed in [16]. In short: The estimation method can be considered to provide accurate data. The proposed biomechanical model can be considered adequate, if the differences found between the two methods are repeatable for one subject. This repeatability will be confirmed by realizing additional experiments.

\section{EXERCISE EVALUATION}

As previously mentioned, exercise evaluation comprises a teach-in mode and a trainer mode (see Figure 1). The aim of the teach-in mode is to obtain for the individual subject and for each exercise a personalized model of one motion cycle, which can serve as reference. The method is to learn this model from correctly performed example executions. For this, motion data (according to the previous section) is collected during the teach-in phase, where the subject is supervised by a physical activity specialist. The model generation from the recorded data is detailed in Section V-A.

The aim of the trainer mode is to control the subject's movements when exercising alone at home. In particular, this means to automatically count the repetitions and to accurately evaluate each repetition based on the respective reference motion. This is explained in Section V-B.

\section{A. Teach-in mode}

This section presents a fully automated method for constructing a reference model from a very short training sequence (see Figure 4). The training data in terms of recorded joint angles is assumed to contain a pre-defined number of exercise repetitions correctly performed by the subject. A Hidden Markov model (HMM) is used to represent the reference model. This representation has been chosen for two reasons: Firstly, it naturally takes variations in motion into account by allowing for time-warping and has thus been successfully applied in domains such as speech, gesture, or handwriting recognition. Secondly, standard algorithms, such as the Viterbi algorithm, can be used for segmentation. The proposed method

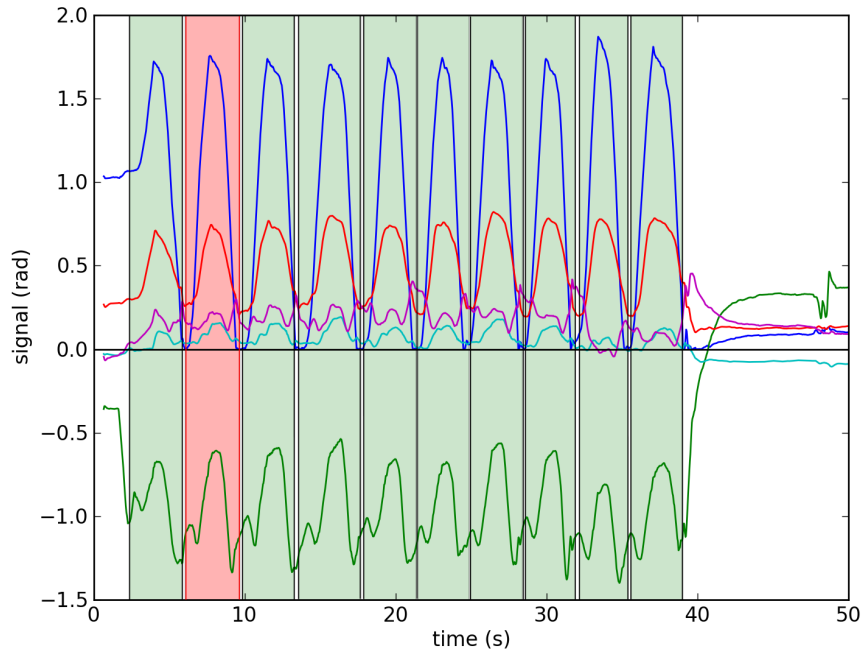

Fig. 4: Teach-in Mode: The best motif candidate (red area) and its occurrences (green areas) in the training signal. The five graphs show the measured joint angles of the right arm during a guided training session.

for model generation consists of two steps: The first step automatically detects candidates for the recurring motion cycles in the training sequence. The second step uses the detected motion cycles to construct the model.

1) Candidate detection: The problem of locating patterns in real-valued, multivariate time series is a known problem and several approaches have been proposed [17], [18]. However, all of these methods are based on a pre-defined window size. Here, the windows size is equivalent to the time needed to execute one exercise repetition. Since the execution time is unknown, the first step consists in estimating a suitable window size. Based on the assumption that the exercise repetitions during the teach-in phase are performed consecutively with roughly the same speed, a dominant frequency will be present in the signal. This can be extracted using the combined power spectral density (PSD) [19]. The window size is then initialized as the wavelength of the dominant frequency. Based on this preprocessing step, an extended version of [18] is then parameterized with the window size to detect the reference motion candidates. The best candidate is evaluated and chosen as final result. Figure 4 illustrates an example of a candidate reference motion cycle and its segmented occurrences in the training signal.

2) Model generation: The observation probabilities of the HMM are modeled as Gaussian mixtures. Here, the different channels (joint angles) are handled separately, so that the system is capable of identifying, in which angle a deviation occurs. The reference motion cycles which have been extracted during the candidate detection are used as training samples for the model. Since traditional parameter estimation methods for HMMs, such as the Baum-Welch algorithm, typically fail when applied to too few training examples, a simple construction algorithm is used to capture the characteristics 


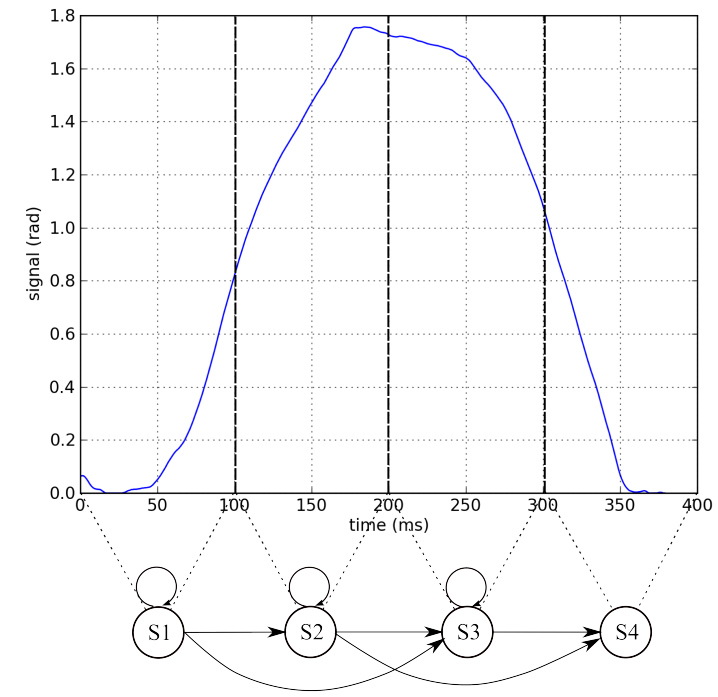

Fig. 5: Personalized Model: HMM for one channel of the signal.

of the reference motion. This algorithm builds a HMM with a left-right topology, which is a wide-spread approach to model time-varying sequential data. Self-transitions and skiptransitions are added to allow for a faster and slower execution of the motion. The number of states is chosen in relation to the estimated window size. Accordingly, each subsequence is divided into equal-length adjacent segments, whereas each segment is assigned to a state. Figure 5 illustrates an example model with only four states. For each state a Gaussian mixture is trained using an expectation-maximization algorithm on all elements of the respective training set. Thus, each segment is described by a normal distribution.

\section{B. Trainer mode}

The trainer mode consists of two phases that build upon each other. First, the reference model is used to detect and segment the repetitions while being performed. During this step, deviations from the reference motion are already identified, but not further characterized. Once, a repetition is completed, the second phase consists in accurately evaluating the quality of the performed motion cycle according to the evaluation criteria introduced below.

1) Motion cycle detection: As already stated, the HMMs obtained during the teach-in mode enable the online detection of the represented reference motion within continuous motion data by means of the Viterbi algorithm. In general, the standard Viterbi algorithm computes the most likely path of states given a sequence of observations. Here, the observations are given by the continuous joint angles as streamed by the data collection component. Thus, the algorithm can determine, to which state, respectively frame, of the reference motion the current motion matches. If the log probability of the Viterbi algorithm is too low, the current observation is considered as noise that is random motion. Whenever a complete motion cycle has been detected, the detailed evaluation starts as described below.
2) Motion cycle evaluation: According to the literature on strength training, in order to ensure safety and effectiveness, it is fundamental to check both the intensity and the technique of exercise. The load of the exercise, the muscles that work but also the posture taken during the exercise have then to be evaluated. Translating these constraints into objective data that are implied in the measurements resulted in the following criteria: For movement load, the exercise intensity is quantified by the number of repetitions, the movement speed, the movement amplitude, and the movement smoothness. For the muscles to work, it was chosen to characterize the axes of rotation. Finally, for safety issues, it was also chosen to characterize the posture by evaluating fixed distances or angles that are recommended when performing the movement. This could, for instance, be the distance between the feet during squat exercises, or the angle at the pelvis during push-ups.

The number of repetitions and their duration are given by the segmentation step described above. For the other criteria, an algorithm has been developed, which evaluates each detected motion cycle using the model constructed during the teach-in phase as reference. The different steps of the algorithms are the following. First, the joint trajectory that has the greatest range of motion during the movement is identified. This range of motion is compared to the reference motion constructed during the teach-in phase. Then, different fixed angles and distances that have to be respected during the movement in order to avoid injuries are computed and compared with those obtained during the movement of reference. Afterwards, the mean rotation axis is computed for the current cycle at each joint based on the quaternion formalism. The mean rotation axes are then compared to the ones obtained during the movement of reference. Using the same formalism, the rotation amplitudes are also compared. Finally, the number of extrema in the derivative of the joint trajectory (i.e. its velocity) that has the greater range of motion during the movement is evaluated and compared in order to determine movement smoothness. The procedure is illustrated in Figure 6. If a deviation greater than a certain percentage is detected in any of the above parameters, the user is informed by an explanatory feedback. After completing the exercise, a summary of the quality is provided by computing the result statistics across all repetitions. This enables detailed feedback after each cycle and a summary at the end.

\section{Implementation and evaluation}

The methods for exercise evaluation are currently implemented in Python and Matlab. Work is in progress to port these to a $\mathrm{C}++$ software component that communicates with both, the data collection software and the data presentation software.

The exercise evaluation has been evaluated in a preliminary study with four subjects. The subjects had to perform three strength training exercises typical in cardiac patients' rehabilitation. In this preliminary study, only upper-extremity exercises were considered, since they generally imply a greater variation in the amplitude and joints to work and they are 


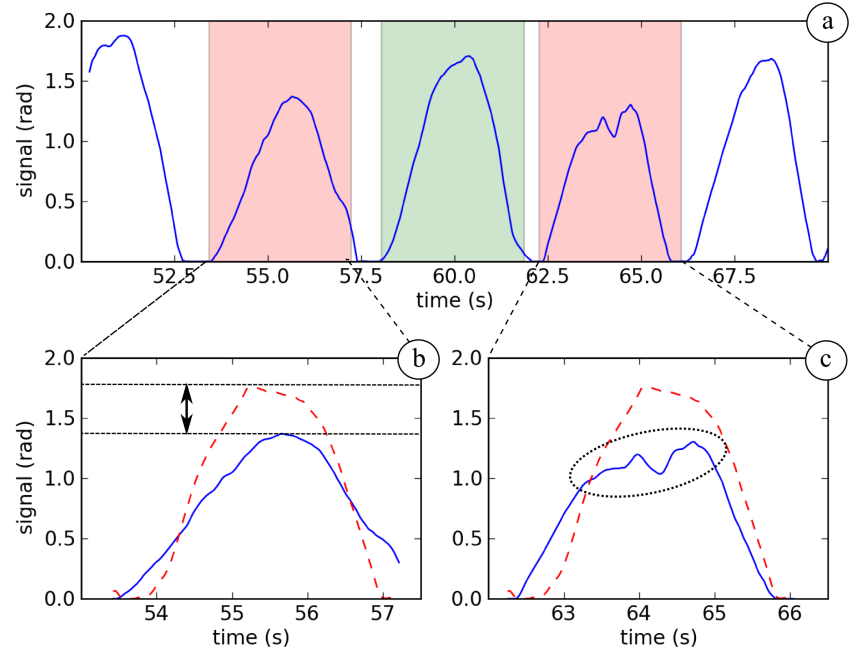

Fig. 6: Training Mode: The personalized model depicted in Figure 5 is used to evaluate the signal of one joint angle (a). The detected motion cycles are evaluated separately. The cycles overlaid with a red area show a significant deviation from the reference movement (illustrated as dashed red lines), either in amplitude (b) or in the number of extrema (c). The green area indicates a correctly performed cycle.

generally less familiar to beginners. These exercises were biceps curls with light weight, overhead press with a stick, and wall push-ups.

The subjects repeated the exercise series four times. The first time, they respected the number of repetitions and the recommendations provided by the rehabilitation program. This corresponds to the teach-in phase. The other times, they modified their posture, the range of motion, the rhythm, and/or the number of repetitions. In order to ensure that the developed algorithms are not material dependent, subject movements were captured by using the motion capture system presented in Section IV, but also the reference setup described in Section IV-C.

The results of applying the developed method to each series of exercise are: The present algorithm was able to identify when the number of series was not respected. For the other parameters, one of the issues was to decide until when the deviation from the reference movement could be considered as being tolerable. With a threshold of ten per cent, the movements that look incorrect to the examiner were all detected as being incorrect by the algorithm. The preliminary tests seem promising but another study including physiotherapists and physical activity teachers will be conducted in order to confirm objectively that the parameters and the thresholds chosen to evaluate the movement are in adequation with their own expertise.

\section{DATA PRESENTATION AND USER INTERFACE}

This section details the data presentation component, i.e. the component that presents the information provided by the other system parts to the user. The focus is on introducing a user interface concept designed for older adults. The challenging problem is: The elderly user should be unaware of the complexity of the underlying software and hardware details. Moreover, the interface should be developed for elderly people with little or no computer experience. The core philosophy is therefore a minimalist design and simplicity of the user interface. The general concept is first introduced and then illustrated in the calibration and exercise mode. The last part of this section describes the implementation of the data presentation component.

\section{A. User interface design}

1) General: One of the most difficult problems in adopting technology is the usage of interfaces that are often not wellsuited for elderly users. Especially, the aspect of technology acceptance needs to be addressed. The technology should aim at being less noticeable than traditional hardware and better integrated with the lifestyle of the elderly [20].

The current set-up for the elderly makes use of a television. The patients are able to enter information interactively via a remote control. With a television being the primary focal point in the everyday lives of the elderly, it is an ideal candidate for a technological aid by embedding intelligence into it [21]. Using the television the need to learn a new interface is negligible, as the user will utilize an already familiar interface. Furthermore, the user is unaware of any underlying software or hardware details within the system, as the monitor visually and behaviorally represents a television with the remote control still being the primary input device.

In addition to the previous described hardware the graphical user interface is the main component with which the user gets into contact. Modern interface design requires the use of memory and sight; all faculties that decrease with age [22]. As people age, their abilities change. This process includes a decline over time in the physical, cognitive and physical functions at different rates relative to each other. Visual changes among aging adults include problems with reading speed, seeing in dim light, reading small print, and locating objects.

For the current system the symbols were designed to be simple and large. A large and clear font was also used in the application and only the most necessary information for use is displayed on the screen (see Figure 7). To support the presented text information a speech output has been introduced for limited vision users. At every point in the interaction it tells the user what he has to do next.

2) Calibration: As indicated in Section IV-B, a valid calibration is important for ensuring repeatable and accurate motion data. Therefore, special work has been undertaken to ensure a valid calibration process. Figure 7 illustrates the step-wise calibration of the upper body. As introduced in Section IV-B, particularly age-related impairments of the musculoskeletal system have been considered for the procedure. The system leads the user through the three-step calibration process. Firstly, the user is asked to stand up straight. For each single step, as mentioned above, text, image, and audio 


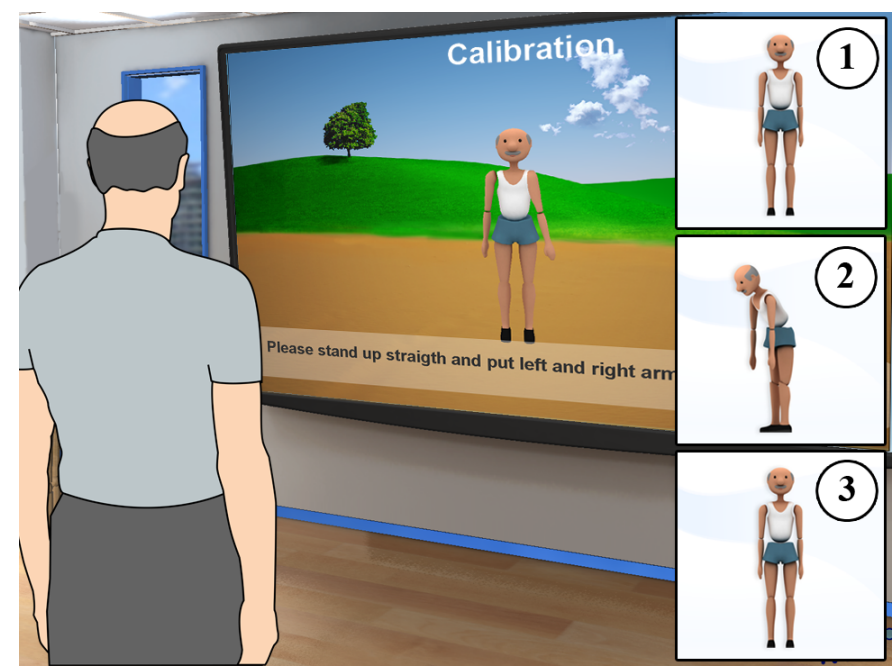

Fig. 7: By means of visual (text and images) and audio instructions the personalized trainer guides the elderly user through the calibration process.

instructions are used to guide the user. With "Please bend forward" the system switches to the next step. Finally, the user is asked to take again an upright body position with the arms straight down and the thumbs forward. When the calibration is completed, the system is ready to visualize the user's movements through his virtual alter ego.

Currently, the calibration step has one limitation: the validity and accuracy of the result is not evaluated after the procedure. In order to tackle this problem, two possible solutions are considered: In case of the sensor jacket, where the positioning of the sensors is roughly fixed, the current calibration can be compared with the previous results. In case of the velcro fixations, it is considered to use an additional reference posture for an interactive check.

3) Exercise: Figure 8 shows the user interfaces in a training mode, e.g. biceps curls. Here, the user only carried out three of five repetitions (see Figure 8a). The system can be used as virtual memory. The application does not require the user to remember how many repetitions he has to perform or he already did. The aim is to avoid causing an excessive memory load on the user. Thus, the elderly user is able to concentrate on the correct execution of the exercise. The digital trainer has the knowledge of the type of exercise, number of repetitions to perform, and successfully carried out repetitions.

Furthermore, the system intervenes when an exercise is performed incorrectly. If the patient moves his shoulder too much a dedicated feedback is displayed on the television (see Figure $8 b$ ). Finally, the system is capable of protecting against overexertion. At certain points during the exercise the system sets a pause (see Figure 8c). Thus, it minimizes the risk of an overuse injury.

\section{B. Implementation and evaluation}

As mentioned in section III, the system is set-up modular and is constructed to act as a client server system. The graphi- cal user interface (client) communicates via a standard TCP/IP connection to the data processing component (server) within the Intranet or the Internet. The communication between client and server is defined by a customized protocol, the Motion Tracking Binary Format (MTBF) protocol, as indicated in section III. The MTBF protocol is designed to only send the necessary information. Therefore, the traffic and amount of data is reduced dynamically. This allows to visualize the data in real-time. The client interprets the MTBF packages correspondingly and displays the information graphically.

Due to the above mentioned aspects it is necessary to implement an application, which is independent of the hardware. The core technology used for the user interface is based on the $\mathrm{Java}^{\mathrm{TM}}$ programming language. Independence, neutrality and portability are the reasons why the visualization component is implemented as a Java ${ }^{\mathrm{TM}}$ Web Start application. This allows for the development of a distributed and platform independent application. The system not only runs on a television setup as described above but also on any personal computer. A physician could make use of the data presentation component to analyze the movements of the patient, for example. To illustrate the movements of the avatar the component exploits the Java3D $^{\mathrm{TM}}$ library. Furthermore, the user interface is designed for different countries and supports multiple languages.

Moreover, an important objective is the maintainability of the content with a minimum maintenance requirement. The Java $^{\mathrm{TM}}$ Web Start technology supports updates of the software. Any changes or updates in the data presentation component will be downloaded automatically. Therefore, the patient does not require any technical expertise and, hence, the need of technological knowledge can be reduced.

First informal evaluations of the user interface have been conducted during several fairs and demonstrations. The feedback has been collected and used to further improve the
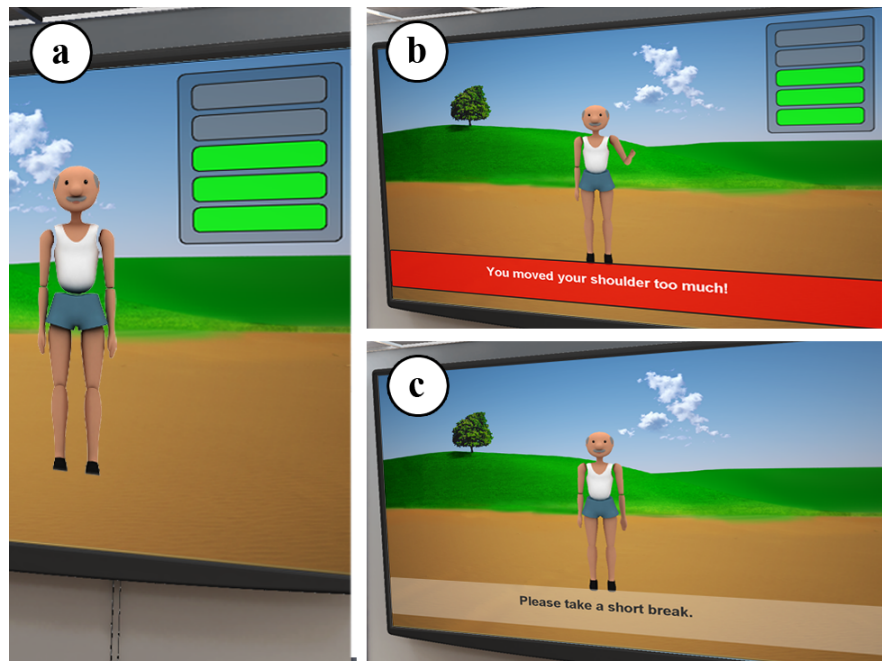

Fig. 8: Interfaces during an exercise: Besides the number of repetitions (a), the system provides feedback in case of wrong execution (b) and protects against overexertion (c). 
system. A formal evaluation of the overall system is the next step.

\section{CONCLUSION AND Future WORK}

This paper outlines a powerful system for supporting the practice of physical activity in clinical settings and in outhospital environments. While providing a short overview of the overall system and its functionalities, the paper then focuses on the special use case of home-based exercise training, more specifically strength exercise monitoring for both prevention or rehabilitation, and in this on the two aspects of personalization and usability for elderly. The first aspect is mainly taken into account in the developed method for exercise evaluation, while the second aspect mainly becomes important in the data presentation, user feedback and interaction.

The system is currently still under development and (technical) evaluations of the individual components have shown promising results as described in the respective sections. The evaluation of the overall system is planned within a clinical assay including 30 subjects, 20 cardiovascular and functional disease patients and 10 fit and healthy subjects, all over 60 years. The strength exercise monitoring use case is planned in supervised sessions at a rehabilitation hospital and a sports medicine unity with about three subjects per day. The goals of the clinical assay will be technical evaluation of the components working together, evaluation of feasibility and usability of the user interfaces, and evaluation of the system in terms of helping the user in his involvement in regular and long term physical activity. The latter two aspects will be based on questionnaires. The clinical assay is at the moment in the application phase and is planned for September 2011 to June 2012 including patient recruiting and data treatment and evaluation.

\section{ACKNOWLEDGMENT}

This work has been performed within the project PAMAP funded under the AAL Joint Programme (AAL-2008-1). The authors would like to thank the project partners, the EU and the national funding authorities for the financial support. Many thanks to Vladimir Hasko for the support in preparing the illustrations. For more information, please visit the website http://www.pamap.org.

\section{REFERENCES}

[1] D. Warburton, C. Nicol, and S. Bredin, "Health benefits of physical activity: the evidence," CMAJ, vol. 174, no. 6, pp. 801-809, 2006.

[2] N. Ashworth, K. Chad, E. Harrison, B. Reeder, and S. Marshall, "Home versus center based physical activity programs in older adults." Cochrane Database Syst Rev, vol. 25, no. 1, 2005.

[3] D. Kirkendall, "Exercise prescription for the healthy adult," Prim Care, vol. 11, no. 1, pp. 23-31, 1984.

[4] M. Rogante, M. Grigioni, D. Cordella, and C. Giacomozzi, "Ten years of telerehabilitation: A literature overview of technologies and clinical applications," NeuroRehabilitation, vol. 27, 2010.
[5] D. Walters, A. Sarela, A. Fairfull, K. Neighbour, C. Cowen, B. Stephens, T. Sellwood, B. Sellwood, M. Steer, M. Aust, R. Francis, C. K. Lee, S. Hoffman, G. Brealey, and M. Karunanithi, "A mobile phone-based care model for outpatient cardiac rehabilitation: the care assessment platform (cap)," BMC Cardiovascular Disorders, vol. 10, 2010.

[6] M. J. Mathie, A. C. F. Coster, N. H. Lovell, and B. G. Celler, "Accelerometry: providing an integrated, practical method for longterm, ambulatory monitoring of human movement," Physiological Measurement, vol. 25, no. 2, p. R1, 2004.

[7] S. I. de Vries, F. G. Garre, L. H. Engbers, V. H. Hildebrandt, and S. van Buuren, "Evaluation of neural networks to identify types of activity using accelerometers," Med Sci Sports Exerc, 2010.

[8] F. W. Dick., Sports training principles. A. \& C. Black, 1997.

[9] A. Reiss and D. Stricker, "Towards Global Aerobic Activity Monitoring," in PETRA'11, 2011.

[10] Trivisio, http://www.trivisio.com.

[11] A. G. Cutti, A. Giovanardi, L. Rocchi, A. Davalli, and R. Sacchetti, "Ambulatory measurement of shoulder and elbow kinematics through inertial and magnetic sensors," Medical and Biological Engineering and Computing, vol. 46, pp. 169-178, 2008.

[12] A. H. Jazwinski, Stochastic Processes and Filtering Theory, ser. Mathematics in Science and Engineering. Academic Press, Inc, 1970, vol. 64 .

[13] A. Reiss, G. Hendeby, G. Bleser, and D. Stricker, "Activity recognition using biomechanical model based pose estimation," in Proc. European Conf. on Smart Sensing and Context (EuroSSC), 2010.

[14] Vicon, http://www.vicon.com/products/viconmx.html.

[15] O. Rettig, L. Fradet, P. Kasten, P. Raiss, and S. I. Wolf, "A new kinematic model of the upper extremity based on functional joint parameter determination for shoulder and elbow," Gait \& Posture, vol. 30, pp. 469-476, 2009.

[16] L. Fradet, A. Reiss, G. Bleser, I. Lamprinos, N. Ville, and O. Machui, "Pamap - system evaluation report," University of Compiegne, Tech. Rep., 2010.

[17] D. Minnen, T. Starner, I. Essa, and C. Isbell, "Discovering characteristic actions from on-body sensor data," in Wearable Computers, 2006 10th IEEE International Symposium on, oct. 2006, pp. 11 -18.

[18] D. Minnen, C. Isbell, I. Essa, and T. Starner, "Discovering multivariate motifs using subsequence density estimation and greedy mixture learning," PROCEEDINGS OF THE, pp. 615-620, 2007.

[19] P. Welch, "The use of fast Fourier transform for the estimation of power spectra: a method based on time averaging over short, modified periodograms," IEEE Transactions on Audio and Electroacoustics, vol. 15, no. 2, pp. 70-73, 1967.

[20] B. Meyer, D. G. Bouwhuis, S. J. Czaja, W. A. Rogers, M. S. Hufschmidt, and J. L. Fozard, "Senior CHI: how can we make technology "elder-friendly?", in CHI '99: CHI '99 extended abstracts on Human factors in computing systems. New York, NY, USA: ACM, 1999, pp. 81-82.

[21] T. T. Hewett, R. Baecker, S. Card, T. Carey, J. Gasen, M. M. G. Perlman, G. Strong, and W. Verplank, "ACM SIGCHI curricula for human-computer interaction,” New York, NY, USA, Tech. Rep., 1992.

[22] M. Zajicek, "Special interface requirements for older adults," in Workshop on Universal Accessibility of Ubiquitous Computing: Providing for the Elderly, 2001. 